\title{
Imaging features and surgery-related outcomes in intraventricular neurocysticercosis
}

\author{
Jonathan Stuart Citow, M.D., J. Patrick Johnson, M.D., Duncan Q. McBride, M.D., \\ AND Mario Ammirati, M.D. \\ Cedars-Sinai Institute for Spinal Disorders, Los Angeles, California; Division of Neurosurgery, \\ Harbor-UCLA Medical Center, Torrance, California; and Section of Neurosurgery, University of \\ Chicago, Chicago, Illinois
}

\begin{abstract}
Object. Neurocysticercosis (NCC) is the most common parasitic infection of the central nervous system, and its prevalence is continuing to increase in the United States. The diagnosis of intraventricular NCC (IVNCC) may be difficult, and surgery frequently fails to resolve symptoms. A retrospective review of magnetic resonance (MR) imaging characteristics and surgery-related outcomes may improve management strategies of this disease.

Methods. The authors report the presentations, neuroimaging characteristics, surgical management, and outcomes of 30 patients with IVNCC treated over a 10-year period (mean follow-up period 4 years). Cysts were located in the lateral ventricles (five cases), the third ventricle (five cases), and the fourth ventricle (21 cases). One patient had lesions in both the lateral and fourth ventricles. Presenting symptoms were related to hydrocephalus or mass effect from the lesions.

All patients underwent computerized tomography (CT) and MR imaging of the brain. Treatment consisted of shunt implantation or primary excision of an IVNCC lesion. Outcomes after operations and reoperations were evaluated in light of enhancement characteristics on MR imaging.

Computerized tomography scanning demonstrated IVNCC lesions in $10 \%$ of cases, and MR revealed lesions in $100 \%$ of cases. In patients in whom gadolinium (Gd) enhancement of IVNCC lesions was demonstrated on MR imaging, the surgery-related failure rate was higher and patients required reoperation, and in those in whom gadolinium enhancement was absent the surgery-related failure rate was lower (64 and 19\%, respectively; $\mathrm{p}<0.0002$ ).

Conclusions. Magnetic resonance imaging is superior to CT scanning for detecting IVNCC lesions. The absence of pericystic Gd enhancement on MR imaging is an indication for excision of the lesions. If pericystic enhancement is present, shunt surgery should be performed, and craniotomy reserved for treatment of those patients with symptomatic lesions secondary to mass effect. A treatment algorithm based on patient symptoms, cyst location, and MR imaging $\mathrm{Gd}$ enhancement characteristics is proposed.
\end{abstract}

\section{KEY WORDS • neurocysticercosis • intraventricular cyst • magnetic resonance imaging}

Worldwide NCC is the most common parasitic infestation affecting the central nervous system. It is the most frequent cause of seizures and hydrocephalus in adults from endemic regions, including Mexico, Central and South America, Asia, Africa, and Eastern Europe. , $^{8,23,30,41}$ Neurocysticercosis has recently become more prevalent in North America, particularly the Southern tier states, because of emigration from endemic regions of the world. ${ }^{17,23,34,39}$

Although NCC is usually self-limited by the life cycle of the parasite, intraventricular involvement is often more difficult to manage because of obstruction of CSF path-

\footnotetext{
Abbreviations used in this paper: $\mathrm{CSF}=$ cerebrospinal fluid; $\mathrm{CT}$ = computerized tomography; $\mathrm{Gd}=$ gadolinium; IVNCC = intraventricular neurocysticercosis; $\mathrm{MR}=$ magnetic resonance; $\mathrm{NCC}=$ neurocysticercosis
}

ways and ependymal inflammation. Brain parenchymal involvement occurs in 60 to $92 \%$ of patients with cysticercosis, but IVNCC occurs in only 7 to $20 \%$ of cases. ${ }^{1,4,16-18,21,23,31,33}$ Neuroimaging diagnosis of IVNCC is often difficult to establish. Although MR imaging is generally accepted as the superior modality, it has not been directly compared with CT scanning in the diagnosis of this disease..$^{42,50}$

The majority of IVNCC lesions are found in the fourth ventricle but can produce mechanical obstruction of CSF flow anywhere in the ventricular system, resulting in hydrocephalus or mass effect-induced focal neurological deficits. $1,19,22,23,42$ Other mechanisms causing symptoms are ependymal inflammation associated with cyst degeneration during the life cycle of NCC or diffuse meningitis causing communicating hydrocephalus, but it is unclear which of these origins causes surgical failures. 
The goals of this study are to correlate the MR imaging characteristics with the surgery-related outcomes in patients with IVNCC and to develop a treatment algorithm for the surgical management based on these findings.

\section{CLINICAL MATERIAL AND METHODS}

\section{Patient Population}

We conducted a retrospective analysis of 30 patients with IVNCC treated at the University of California at Los Angeles-affiliated hospitals during a 10 -year period between 1987 and 1996. Follow-up duration ranged from 2 to 11 years (mean 4 years). Twenty-nine patients were Hispanic and one was Asian. Twenty patients were female and 10 were male. Ages ranged from 14 to 71 years (mean 36 years).

\section{Presenting Symptoms and Diagnostic Studies}

The presenting IVNCC-related signs and symptoms are listed in Table 1. As an initial evaluation, all patients underwent CT scanning of the brain. Those patients with acute obstructive hydrocephalus underwent temporary external ventricular drainage until definitive diagnosis and therapy were rendered. All patients subsequently underwent MR imaging of the brain, with and without administration of intravenous $\mathrm{Gd}$. The clinical diagnosis of $\mathrm{NCC}$ was determined based on the patient's history, presenting signs and symptoms, and CT and MR imaging findings. In those patients in whom the diagnosis remained unclear, CSF and serum NCC antibody assays were obtained.

\section{Surgical Management}

Patients underwent one of the following therapies: 1) craniotomy and lesion excision, 2) placement of a ventricular shunt; or 3) craniotomy, lesion excision, and delayed placement of a ventricular shunt for recurrent or persistent hydrocephalus.

Patients with cysts causing hydrocephalus that were located in a surgically accessible region underwent craniotomy and excision of the lesion. Lateral and third ven-

TABLE 1

Presenting signs and symptoms in 30 patients with IVNCC

\begin{tabular}{lc}
\hline \multicolumn{1}{c}{ Signs/Symptom } & No. of Cases \\
\hline headache & 23 \\
nausea \& vomiting & 19 \\
altered sensorium & 8 \\
ataxia & 8 \\
papilledema & 6 \\
diplopia & 5 \\
blurry vision & 3 \\
vertical gaze paresis & 3 \\
vertigo & 3 \\
nystagmus & 3 \\
sixth nerve palsy & 2 \\
decreased visual acuity & 2 \\
seizure & 2 \\
memory disturbance & 1 \\
spasticity & 1 \\
\hline
\end{tabular}

tricular cysts were excised by a frontal craniotomy via a transcortical exposure because of ventricular dilation. Resection of fourth ventricular lesions was performed via a midline suboccipital craniotomy and transvermian exposure. Craniotomy was undertaken in all patients harboring a lesion that caused symptoms due to mass effect. Patients with hydrocephalus and lesions that were difficult to treat surgically underwent shunt surgery, which was from the right frontal lateral ventricle to the peritoneal cavity, unless otherwise required due to the presence of an isolated enlarged ventricle.

\section{Clinical Review and MR Imaging Characteristics}

A retrospective review of all clinical and neuroimaging data was performed in all patients with IVNCC. We used the CT and MR imaging interpretations rendered by the staff neuroradiologist when each patient underwent his/ her initial and subsequent imaging studies. The surgical procedures were reviewed from hospital records. We then conducted the following: 1) comparison of CT and MR imaging features to determine neuroimaging diagnosis of IVNCC; 2) analysis of whether lesion excision or ventricular shunt surgery was a more effective treatment; and 3 ) determination of whether the presence (or absence) of Gd enhancement surrounding an IVNCC lesion on preoperative MR imaging was predictive of successful lesion excision compared with shunt surgery.

\section{Statistical Analysis}

In the statistical analysis for comparison of the aforementioned data we used the Bernoli process. ${ }^{15}$ This allows for evaluation of percentage comparisons between different groups based on diagnostic or therapeutic regimens. A probability value of 0.05 was used as the critical value for statistical significance for the entire study.

\section{RESULTS}

\section{Symptoms and Intraventricular Cyst Location}

The most common IVNCC-related presenting symptoms were headache and nausea/vomiting (Table 1). Other presenting symptoms were comparatively infrequent. Many patients experienced more than one symptom, as noted in Table 1. Presenting symptoms related to hydrocephalus were the most common. The location of IVNCC lesions are summarized in Table 2.

\section{Comparison of Neuroimaging Modalities}

Computerized tomography scanning revealed IVNCC lesions in three of 30 patients, and MR imaging demon-

TABLE 2

Location of IVNCC lesions

\begin{tabular}{cc}
\hline \hline Localization & No. of Cases \\
\hline lat ventricles & $5^{*}$ \\
3rd ventricle & 5 \\
4th ventricle & $21^{*}$ \\
\hline
\end{tabular}

* One patient harbored cysts in the lateral and fourth ventricles. 
strated the intraventricular lesions in all 30 patients $(\mathrm{p}<$ 0.001) (Figs. 1-3). In patients in whom CT scanning clearly identified intraventricular lesions there was evidence of a high density lesion (that is, probable calcified scolex) with ventricular dilation proximal to the lesion (Figs. $1 \&$ 2). Notably, parenchymal calcifications, which are common findings in patients with parenchymal NCC, were identified by CT scan in $15(50 \%)$ of 30 patients. Ventricular enlargement due to CSF obstruction was the most common neuroimaging finding in 29 patients (97\%).

\section{Surgical Management}

Lateral Ventricular NCC. There were five patients with IVNCC lesions in the lateral ventricles (Table 2), and all presented with hydrocephalus. Three patients underwent shunt surgery as a primary procedure, and two required no further treatment (Table 3). One of these patients underwent suboccipital craniotomy to remove a symptomatic fourth ventricular lesion, subsequently required multiple shunt revisions for obstruction, and ultimately died of complications related to brain edema, aseptic meningitis, and repeated shunt failure. Two patients underwent craniotomy and resection, and no further treatment (that is, no shunt surgery) was required.

Third Ventricular NCC. There were five patients with third ventricular cysts, and all presented with hydrocephalus. In four a shunt was placed, and in one craniotomy was performed to allow removal of the lesion (primary procedures) (Table 3). In the patient who required craniotomy and cyst excision mass effect in the ventricle caused hemiparesis that resolved postoperatively. None of the patients with third ventricular lesions required further therapy regardless of treatment (that is, shunt surgery or craniotomy).

Fourth Ventricular NCC. There were 21 patients with fourth ventricular cysts. Twenty of the patients presented with hydrocephalus and one with mass effect (brainstem compression with quadriparesis and bilateral sixth cranial nerve palsies). In 11 patients shunts were placed, and 10 underwent craniotomy and lesion excision (Table 3). Of the 11 shunt-treated patients, two required no further treatment, four required shunt revision, and five underwent subsequent craniotomy for cyst excision. Of the 10 patients in whom initial craniotomy was performed, three required subsequent placement of a shunt for recurrent or persistent hydrocephalus.

The overall reoperation rate in patients who underwent initial shunt surgery was $61 \%$ and in those who underwent initial craniotomy, the reoperation rate was $23 \%$ (Table 3 ). Despite a numerical difference, they were not statistically different $(\mathrm{p}>0.05)$.

\section{Magnetic Resonance Imaging Enhancement and Outcome}

On MR imaging, Gd enhancement surrounding the IVNCC lesions was present in 14 patients and absent in 17 patients (the patient with both lateral and fourth ventricular cysts being counted twice) (Table 4). There were no differences in clinical symptoms or presentation between these groups.

Lateral Ventricles. Of the five patients with lateral ventricular cysts, Gd enhancement was demonstrated in three and absent in two (Table 4). One patient in whom Gd enhancement was present required multiple shunt revisions and a subsequent craniotomy for excision of a fourth ventricular lesion; this patient later died of complications related to shunt malfunction. The other two patients in whom MR imaging revealed Gd enhancement underwent placement of a shunt and required no further surgical treatment. The two patients in whom MR imaging demonstrated no $\mathrm{Gd}$ enhancement required no further surgical treatment after the lesion was resected.

Third Ventricle. There was one patient in whom MR imaging revealed $\mathrm{Gd}$ enhancement and four in whom none was demonstrated (Table 4). None of these patients required further surgical treatment regardless of initial treatment (that is, shunt surgery or craniotomy).

Fourth Ventricle. Magnetic resonance imaging revealed $\mathrm{Gd}$ enhancement of the fourth ventricle in 10

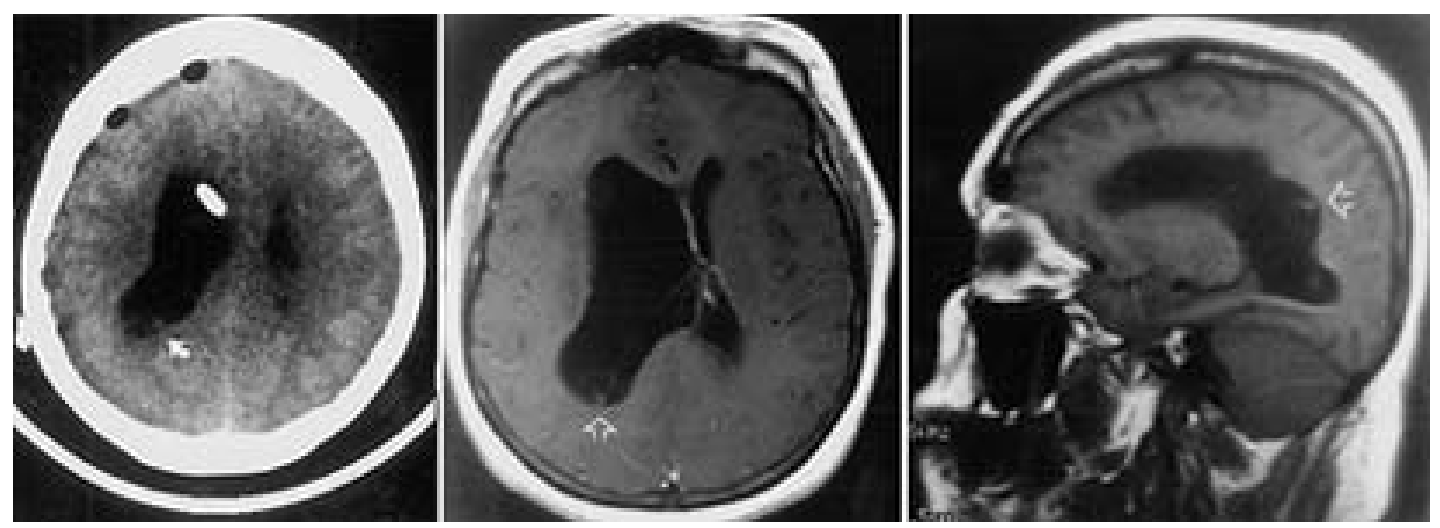

Fig. 1. Neuroimaging studies obtained in a 40-year-old man who presented with headache, nausea, and mild left hemiparesis and was found to have right lateral ventricular enlargement. Left: Axial CT scan poorly demonstrates lesion in right trigone region (curved arrow). Center: Axial $\mathrm{T}_{1}$-weighted MR image revealing a minimally enhancing cystic lesion (open arrow) with probable scolex. Right: Sagittal MR image demonstrating the lesion (open arrow). He was treated initially with external ventricular drainage, then underwent craniotomy and cyst excision. He subsequently required placement of a ventriculoperitoneal shunt. 

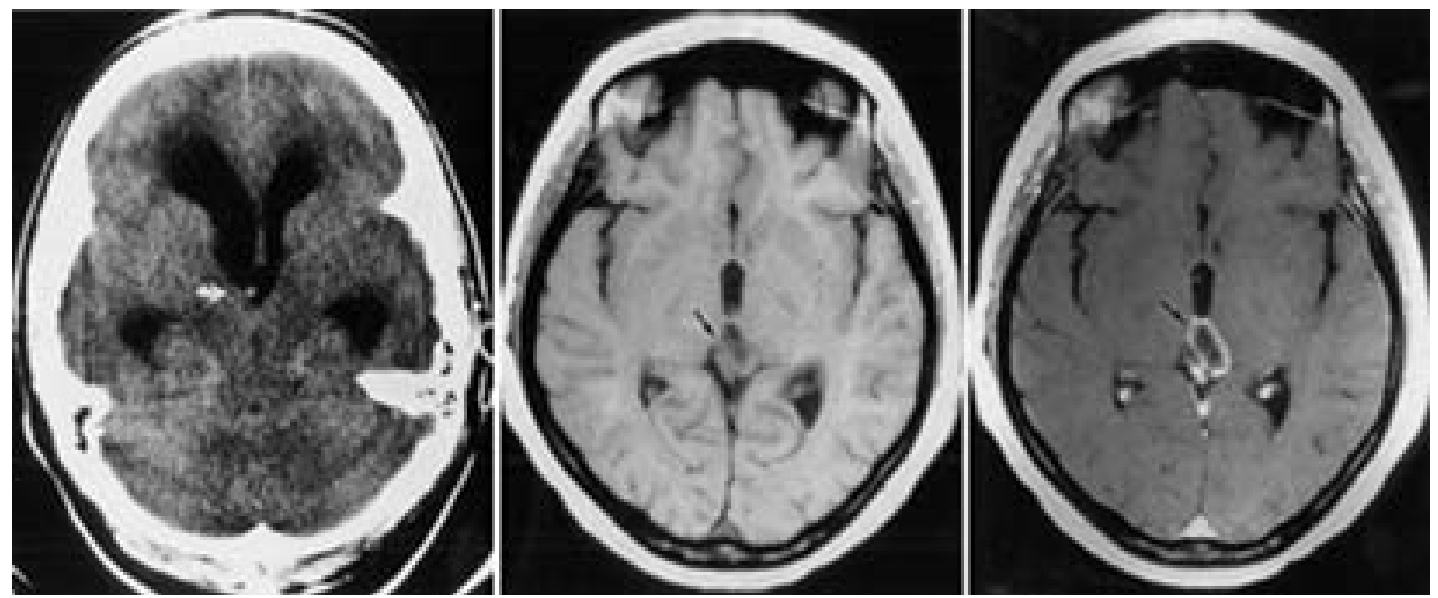

Fig. 2. Neuroimaging studies obtained in a 28 -year-old Hispanic woman who presented with headache, nausea, and vomiting and was found to have obstructive hydrocephalus due to a third ventricular lesion. Left: Axial CT scan demonstrating a calcified lesion (presumed scolex) in the anterior third ventricle (open arrow). Center and Right: Axial $\mathrm{T}_{1}$-weighted MR images demonstrating the lesion and cystic mass (arrows) in the third ventricle (center) with $\mathrm{Gd}$ enhancement (right). She underwent placement of a ventriculoperitoneal shunt and no further treatment was necessary.

patients and no enhancement in 11 patients. Reoperation was required in eight patients with IVNCC-related Gd enhancement, and in six cases these were shunt revisions. Of the 11 patients in whom neuroimaging revealed no $\mathrm{Gd}$ enhancement, three required reoperations.

The overall reoperation rate for patients in whom $\mathrm{Gd}$ enhancement was present was $64 \%$ compared with $19 \%$ in those patients in whom enhancement was absent $(\mathrm{p}<$ $0.00025)$.

\section{DISCUSSION}

\section{Summary of Findings}

Analysis of our data suggests three conclusions: 1) MR imaging is significantly better than CT scanning in the di- agnosis of IVNCC; 2) in cases of nonenhancing IVNCC, craniotomy (open or endoscopic) should be performed and the cyst excised; and 3) in cases of enhancing lesions patients should probably undergo placement of a shunt. The presence of enhancement on MR imaging likely denotes ependymal and leptomeningeal inflammation; hence, even after cyst excision, hydrocephalus is likely to develop.

\section{Overview of Cysticercosis}

The tapeworm Taenia solium has a unique life cycle in which humans are the definitive host and pigs are the intermediate host. ${ }^{22,29}$ The disease manifests as two distinct clinical entities: taeniasis and cysticercosis. Taeniasis results from the ingestion of undercooked pork containing
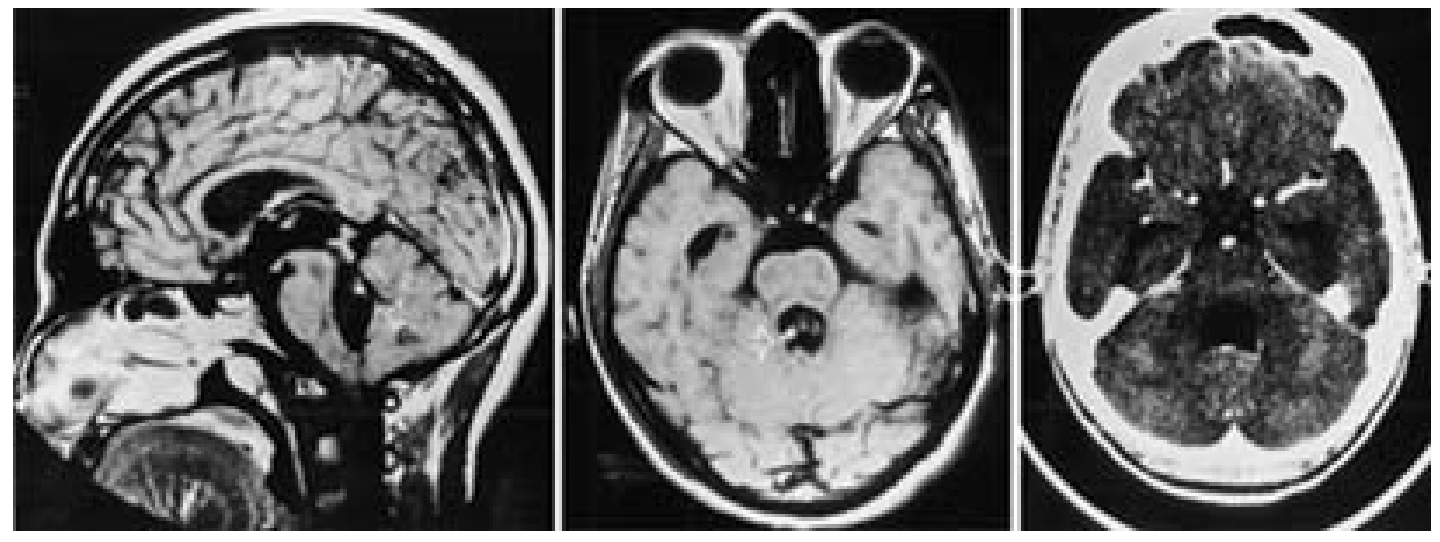

Fig. 3. Neuroimaging studies obtained in a 26-year-old Hispanic man who presented with headache, diplopia, and ataxic gait. Left: Axial CT scan demonstrating marked enlargement of all ventricles consistent with obstructive hydrocephalus but failing to identify a fourth ventricular lesion. Center: Axial nonenhanced $\mathrm{T}_{1}$-weighted MR image clearly revealing a probable scolex in the cephalad aspect of the fourth ventricle (open arrow). Right: Sagittal MR image demonstrating adjacent gadolinium enhancement. The patient underwent suboccipital craniectomy and excision of the lesion but later required shunt implantation to treat recurrent ventriculomegaly. 
Intraventricular neurocysticercosis

TABLE 3

Summary of surgical management

\begin{tabular}{lcccc}
\hline \hline & \multicolumn{4}{c}{ Ventricles } \\
\cline { 2 - 5 } & Lateral & Third & Fourth & Total No. \\
\hline no. of cases & 5 & 5 & 21 & 31 \\
initial shunt group & 3 & 4 & 11 & 18 \\
shunt revision(s) & $1^{*}$ & 0 & 4 & $5 \dagger$ \\
subsequent craniotomy & $1^{*}$ & 0 & 5 & $6 \dagger$ \\
initial craniotomy group & 2 & 1 & 10 & 13 \\
subsequent shunt & 0 & 0 & 3 & $3 \dagger$ \\
\hline
\end{tabular}

* The same patient harbored both lateral and fourth ventricular lesions and required both multiple shunt revisions as well as suboccipital excision of the fourth ventricular lesion.

$\dagger$ Reoperation rates in patients who initially underwent shunt surgery (11 [61\%] of 18$)$ and those who initially underwent craniotomy (3 [23\%] of 13) were not statistically different $(\mathrm{p}>0.05)$.

the mature cysticercus of $T$. solium, which develops into the adult worm in the human jejunum. The tapeworm is composed of a scolex and multiple egg-containing proglottids that periodically detach from the tapeworm and are excreted in the human stool. The eggs are then ingested by pigs and the cycle may be repeated, representing a relatively benign form of this disease. ${ }^{29,46}$

Cysticercosis in humans is acquired by ingestion of eggs containing primary larvae of T. solium. ${ }^{12}$ Gastric acid activates the eggs to hatch, which releases the larvae into the upper small bowel where they penetrate the intestinal mucosa to enter the systemic circulation and disperse throughout the bloodstream. Human infestation with the primary larvae can occur in one of three fashions: 1) heteroinfestation (by ingestion of food or water contaminated by other human feces); 2) external self-infestation (ingestion of one's own contaminated stool); or 3) internal self-infestation (reverse peristalsis transporting the eggs from the small intestine into the stomach where they may hatch).

\section{Overview of NCC}

The larvae of T. solium have a marked predilection for the skin, muscle, and the central nervous system. ${ }^{2,6} \mathrm{Neu}-$ rocysticerosis results from hemopoietic dissemination of the larvae to the meninges, brain parenchyma, or ventricular system. The brain is reported to be involved in 60 to $92 \%$ of cases, and in 7 to $20 \%$ of these cases the ventricular system is primarily involved. ., $^{1,18,21,23,33}$ Brain parenchyma and intraventricular lesions develop by migration of the primary larvae through the capillaries and choroid plexus, respectively. The primary larva develops into secondary larva, or a cysticercus, with a scolex in a fluidfilled bladder within 60 to 70 days. ${ }^{12}$ The parenchymal lesions remain localized, but intraventricular lesions can percolate through the ventricular system toward the fourth ventricle where most are found. . $^{1,20,39}$ The migration through the ventricular system has been well documented and underscores the need for conducting a neuroimaging study prior to the planned excision to confirm that the lesion location has not changed. ${ }^{1}$

The cysticercus lesions occur in different forms in the human brain. ${ }^{11,24}$ Cysticercus cellulosae is a thin-walled cyst measuring 4 to $20 \mathrm{~mm}$ in diameter, with a round 2- to
TABLE 4

Summary of MR imaging enhancement stratified by location of the cyst

\begin{tabular}{ccc}
\hline \hline & \multicolumn{2}{c}{ MR Enhancement } \\
\cline { 2 - 3 } Location & Present $(14$ cases $)$ & Absent $(17$ cases $)$ \\
\hline lat ventricles & $3^{*}$ & 2 \\
reop $\dagger$ & 1 & 0 \\
3 rd ventricle & 1 & 4 \\
reop $\dagger$ & 0 & 0 \\
4th ventricle & $10^{*}$ & 11 \\
reop $\dagger$ & 8 & 3 \\
total reops & $9(64 \%) \ddagger$ & $3(18 \%) \ddagger$ \\
\hline
\end{tabular}

* One patient harbored lateral and fourth ventricular lesions and underwent shunt surgery and craniotomy.

$\dagger$ Either shunt revision or craniotomy.

$\$$ Reoperation rate based on comparison of presence or absence of $\mathrm{Gd}$ enhancement $(\mathrm{p}<0.00025)$

3-mm mural nodule, which is an immature scolex considered viable and usually detected in brain parenchyma or in

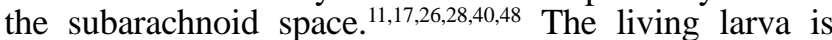
thought to secrete factors that actively suppress the host immune system, producing only a mild inflammatory response. The parenchymal lesions have a terminal lifespan and larval degeneration activates the host immune system resulting in the greatest inflammation that is associated with the racemose form of cysticercosis. ${ }^{28}$ This latter form tends to be a multilobular grape-like cluster several centimeters in diameter and is most frequently found in the basal cisterns, sylvian fissure, or ventricular system. This form lacks a scolex and is considered to be a sterile degenerated remnant of $C$. cellulosae. As the cyst undergoes further degeneration, it is usually visualized as a calcified nodule in brain parenchyma. ${ }^{11,17,26,28,40,48}$ Cysts in these differing forms may be detected in the same patient by CT and/or MR imaging, and the number ranges from a solitary lesion to thousands of lesions, although fewer than 10 per patient are usually detected. .11,17,26,28,40,48 $^{-1}$

\section{Clinical Diagnosis}

The diagnosis of IVNCC is made based on a combination of clinical and demographic data, symptoms, neuroimaging characteristics, and, if needed, results of laboratory tests. ${ }^{2,3,531,40}$ Neurocysticercosis is commonly found in people from endemic third-world countries and is the most frequent cause of seizures and hydrocephalus in adults from Mexico, Central and South America, Asia, Africa, and Eastern Europe., 8,23,30,38

Brain parenchymal involvement is the most common clinically significant manifestation and occurs in 30 to $63 \%$ of cases presenting as seizures, headaches, altered sensorium, or focal neurological signs. ${ }^{17,18,23,31,33,37,43}$ Intraventricular NCC is uncommon by comparison and usually produces symptoms related to CSF obstruction, although it can also cause symptoms from mass effect or ependymal inflammation. , $^{8,917,18,23,31,33,35-37,43}$ A combination of these obstructive and inflammatory symptoms occurs in $23 \%$ of cases. ${ }^{8,9,35,36}$ Mobile deformable lesions may cause intermittent or positional CSF obstruction with episodic elevations of intracranial pressure due to a ballvalve mechanism (Brun syndrome), causing life-threaten- 
ing acute obstructive hydrocephalus. ${ }^{35,51}$ Ependymal and arachnoidal inflammation can also cause obstruction of subarachnoid pathways and subsequent hydrocephalus. Diffuse meningeal inflammation has been reported to occur in 27 to $56 \%$ of cases, usually manifesting with headache, nausea, emesis, nuchal rigidity, and photophobia. ${ }^{17,18,23,31,33,37,43}$ Similarly, this can also result in diffuse basilar arachnoiditis that produces arteritis, cranial neuropathies, or hydrocephalus.

\section{Comparison of Neuroimaging Modalities}

The neuroimaging diagnosis of IVNCC in this series underscored the efficacy of MR imaging over CT scanning. Routine CT scanning is excellent for identification of parenchymal calcifications, which are present in nearly $50 \%$ of patients with NCC, but is limited in the detection of intraventricular lesions because they often appear isodense with CSF and may conform to the shape of the ventricle. ${ }^{32,42,50}$ Although MR imaging has been described as superior for detection of cisternal and IVNCC lesions in several reports, a direct comparison between the two modalities has not been published. Computerized tomography ventriculography has also been useful in our experience for detecting intraventricular lesions, but it requires an invasive procedure and may be limited by visualization only of the ventricular system proximal to an obstructing lesion. ${ }^{21,49}$

On several imaging sequences, particularly on proton density sequences, MR imaging clearly demonstrates IVNCC lesions with a cyst wall and probable scolex from the slightly lower-intensity CSF and higher-intensity fluid in the cyst. Neurocysticercosis lesions are also more easily differentiated because they degenerate, presumably due to the higher protein content within the cyst. ${ }^{42,50} \mathrm{~A}$ scolex, when present, is most clearly identified on $\mathrm{T}_{2}$-weighted images on which the lower-intensity signal of the scolex is distinguished from the high-intensity cystic fluid. ${ }^{42,50}$

\section{Laboratory Diagnosis}

The following laboratory investigations for NCC can be a useful adjunct: CSF evaluation of peripheral blood smears, stool examination, and immunological studies. Cerebrospinal fluid abnormalities of lymphocytic pleocytosis, elevated protein, and hypoglycorrhachia are not sensitive, nonspecific, and have minimal diagnostic value. ${ }^{17,23,37,43,47}$ Eosinophilia occurs in only $20 \%$ of peripheral blood smears. ${ }^{11,17,27}$ Taenia solium ova in stool examinations are found in only $4 \%$ of cases, and their presence indicates concomitant taeniasis infestation and not NCC. ${ }^{17,23,25}$

Serological studies of CSF and serum with enzymelinked immunosorbent assay and enzyme-linked immunoelectrotransfer blot are useful when clinical diagnosis is unclear. The former assay is associated with serum and CSF sensitivities ranging from 65 to $87 \%$ and 62 to $90 \%$, respectively, for NCC and is slightly higher for IVNCC; $2,7,10,13$ its specificity in serum is 63 to $100 \%$ and in CSF it is 98 to $100 \% .^{2,7,10,13,45}$ The sensitivity of enzymelinked immunoelectrotranfer blot serum and CSF ranges from 94 to $100 \%$ and its specificity is $100 \% .^{10,14,44,46} \mathrm{Nev}-$ ertheless, neither of these tests can differentiate NCC from intestinal Taeniasis and must be correlated with the clinical and neuroimaging data. ${ }^{2}$

\section{Surgical Management}

Surgical management of IVNCC is problematic because of the high rates of reoperation primarily related to

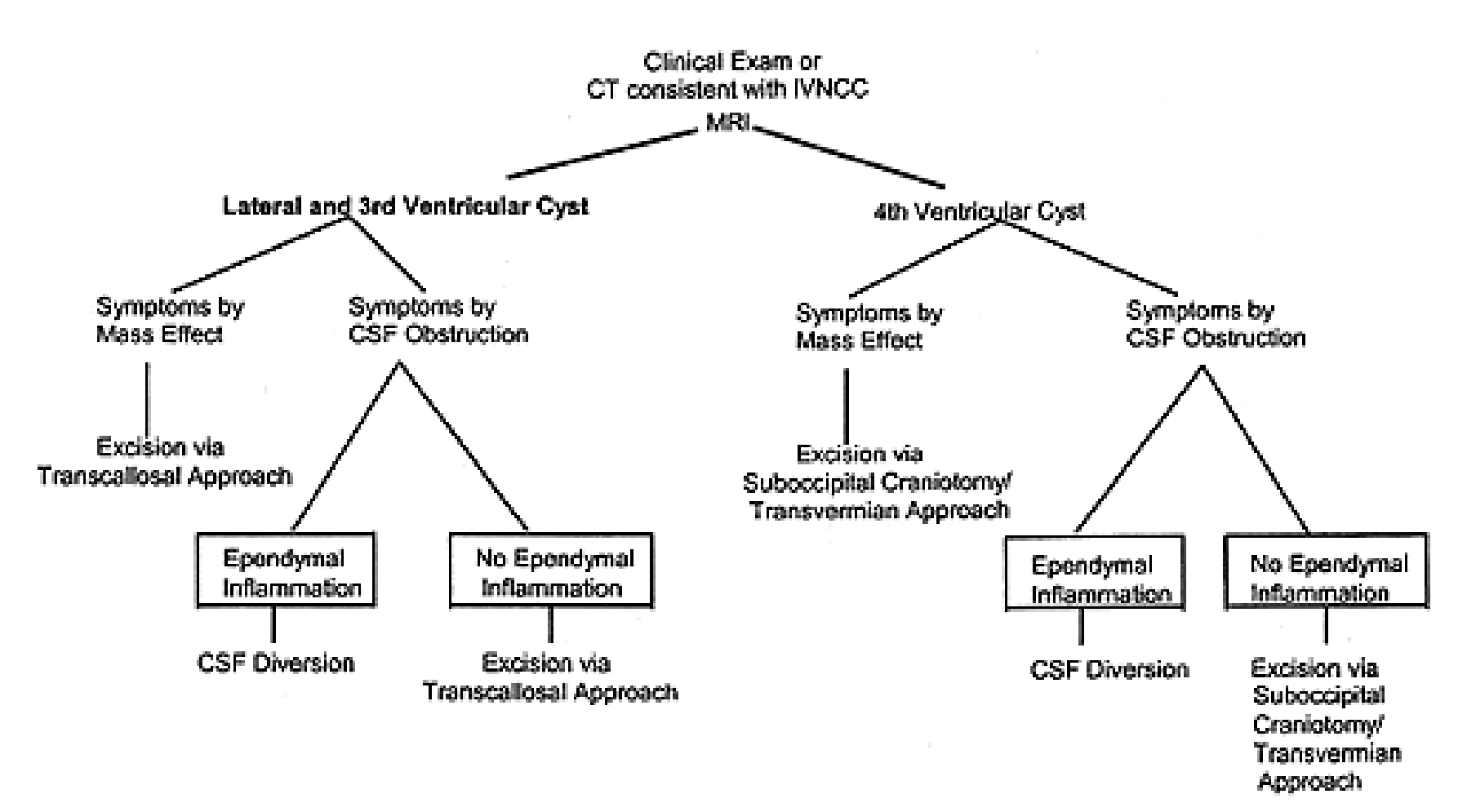

Fig. 4. Management algorithm depicting treatment of patients with IVNCC. 
hydrocephalus after cyst excision or shunt failures, and because there are a few existing guidelines. ${ }^{1,19,20,21,39}$ Previous reports of the surgical management for IVNCC were published during the CT era, and no large series of surgery-related outcomes has yet been published since the advent of MR imaging. ${ }^{1,49}$ Analysis of our data suggests that the reoperation rate after shunt surgery is higher than craniotomy/excision (Table 3). Although the differences in those patients who underwent initial placement of a shunt (11 [61\%] of 18 compared with craniotomy) three [23\%] of 13 did not reach statistical significance $(\mathrm{p}>$ 0.05 ), the percentages are compelling. Although shunt implantation is technically less demanding, recurrent obstruction-related problems potentially would be avoided if a craniotomy/excision alone were successful. ${ }^{1,19} \mathrm{Nev}-$ ertheless, some patients may require subsequent shunt surgery despite successful craniotomy/excision, which occurred in nearly $25 \%$ of the patients we treated. Alternatively, the reoperation rate was nearly twofold higher in patients who initially underwent shunt placement; these reoperations included shunt revision or craniotomy.

Gadolinium enhancement of IVNCC lesions on MR scanning was an indicator of the poor treatment efficacy of these cysts, regardless of the procedure performed (Table 4). The presence or absence of enhancement can thus be used to create an algorithm for the management of IVNCC lesions (Fig. 4). Enhancement presumably indicates the presence of diffuse ependymal inflammation, representing the greater difficulty required to resect these lesions because of arachnoidal and ependymal scarring. ${ }^{1,19,49}$ Therefore, patients in whom MR imaging reveals enhancing IVNCC lesions may be preferably treated with a shunt procedure, and those in whom this modality demonstrates no enhancing IVNCC lesions should be considered for craniotomy and lesion excision because the risk that secondary shunt surgery will be required, based on this experience, is lower.

The future management of intraventricular lesions will continue to evolve as new minimally invasive endoscopic surgery begins to replace open craniotomy in certain cases and as third ventriculostomy may reduce the need for shunt surgery in some patients with obstructive hydrocephalus. This technology will also allow further evaluation of the issues regarding the presence (or absence) of Gd enhancement and surgical treatment options.

\section{CONCLUSIONS}

Intraventricular NCC lesions produce symptoms by obstruction of CSF pathways, and most are found in the fourth ventricle to which they tend to migrate. Magnetic resonance imaging clearly demonstrates these poorly defined lesions, and the presence of Gd enhancement is associated with a higher rate of treatment failure requiring reoperation. If Gd enhancement is absent on MR imaging, craniotomy combined with cyst excision is the preferred initial treatment, and if enhancement is present shunt surgery is possibly the preferred treatment.

\section{Acknowledgments}

The authors thank David Hovda, Ph.D., for his assistance with statistical analysis and Barbara Staton for her editorial assistance. We also thank W. Eugene Stern, M.D., for his advice on the preparation of this manuscript.

\section{References}

1. Appuzo MLJ, Dobkin WR, Zee CS, et al: Surgical considerations in treatment of intraventricular cysticercosis. An analysis of 45 cases. J Neurosurg 60:400-407, 1984

2. Barry M, Kaldjian LC: Neurocysticercosis. Semin Neurol 13: 131-143, 1993

3. Brailsford JF: Cysticercus cellulosae-its radiographic detection in the musculature and central nervous system. Br J Radiol 14:79-93, 1941

4. Brown WJ, Voge M: Neuropathology of Parasitic Infections. New York, Oxford University Press, 1982

5. Cárdenas y Cárdenas J: II. Pathologic and radiologic findings. J Neurosurg 19:635-640, 1962

6. Colli BO, Martelli N, Assirati JA Jr, et al: Results of surgical treatment of neurocysticercosis in 69 cases. J Neurosurg 65:309-315, 1986

7. Corona T, Pascoe D, Gonzalez-Barranco D, et al: Anticysticercous antibodies in serum and cerebrospinal fluid in patients with cerebral cysticercosis. J Neurol Neurosurgy Psychiatry 49:1044-1049, 1986

8. Del Brutto OH, Santibanez R, Noboa CA, et al: Epilepsy due to neurocysticercosis: analysis of 203 patients. Neurology 42: 389-392, 1992

9. Del Brutto OH, Sotelo J: Neurocysticercosis: an update. Rev Infect Dis 10:1075-1087, 1988

10. Diaz JF, Verastegui M, Gilman RH, et al: Immunodiagnosis of human cysticercosis (Taenia solium): a field comparison of an antibody-enzyme-linked immunosorbent assay (ELISA), an antigen-ELISA, and an enzyme-linked immunoelectrotransfer blot (EITB) assay in Peru. The Cysticercosis Working Group in Peru (CWG). Am J Trop Med Hyg 46:610-615, 1992

11. Dixon HBF, Lipscomb FM: Cysticercosis: An Analysis and Follow-Up of 450 Cases. London: H. M. Stationary Office, 1961

12. Escobar A: The pathology of neurocysticercosis, in Palacios E, Rodriguez-Carbajal J, Taveras JM (eds): Cysticercosis of the Central Nervous System. Springfield, IL: Charles C Thomas, 1983, pp 27-54

13. Espinoza B, Ruiz-Palacios G, Tovar A, et al: Characterization by enzyme-linked immunosorbent assay of the humoral immune response in patients with neurocysticercosis and its application in immunodiagnosis. J Clin Microbiol 24:536-541, 1986

14. Feldman M, Plancarte A, Sandoval M, et al: Comparison of two assays (EIA and EITB) and two samples (saliva and serum) for the diagnosis of neurocysticercosis. Trans R Soc Trop Med Hyg 84:559-562, 1990

15. Hayes WL: Statistics for Social Sciences, ed 2. New York: Holt, Rinehart, and Winston, 1973, pp 177-188

16. Leblanc R, Knowles KF, Melanson D, et al: Neurocysticercosis: surgical and medical managment with praziquantel. Neurosurgery 18:419-427, 1986

17. Loo L, Braude A: Cerebral cysticercosis in San Diego. A report of 23 cases and a review of the literature. Medicine 61: 341-359, 1982

18. Lopez-Hernandez A, Garaizar C: Childhood cerebral cysticercosis: clinical factors and computed tomographic finding in 89 Mexican children. Can J Neurol Sci 9:401-407, 1982

19. Loyo M, Kleriga E, Estanol B: Fourth ventricular cysticercosis. Neurosurgery 7:456-458, 1980

20. Madrazo I, Garcia-Renteria JA, Sandoval M, et al: Intraventricular cysticercosis. Neurosurgery 12:148-152, 1983

21. Madrazo I, Renteria JAC, Paredes G, et al: Diagnosis of intraventricular and cisternal cysticercosis by computerized tomog- 
raphy with positive intraventricular contrast medium. J Neurosurg 55:947-951, 1981

22. Marquez-Monter H: Cysticercosis, in Marcial-Rojas R (ed): Pathology of Protozoal and Helminthic Diseases With Clinical Correlation. Baltimore: Williams \& Wilkins, 1971, pp 592-617

23. McCormick GF, Zee CS, Heiden J: Cysticercosis cerebri. Review of 127 cases. Arch Neurol 39:534-539, 1982

24. Miller B, Grinnell V, Goldberg MA, et al: Spontaneous radiographic disappearance of cerebral cysticercosis: three cases. Neurology 33:1377-1379, 1983

25. Mitchell WG, Crawford TO: Intraparenchymal cerebral cysticercosis in children: diagnosis and treatment. Pediatrics 82: 76-82, 1988

26. Nash TE, Neva FA: Recent advances in the diagnosis and treatment of cerebral cysticersosis. N Engl J Med 311:1492-1496, 1984

27. Nieto O: Cysticercosis of the nervous system: diagnosis by means of spinal fluid complement fixation test. Neurology 6: 725-738, 1956

28. Rabiela-Cervantes MT, Rivas-Hernandez A, Rodriguez-Ibarra J, et al: Anatomopathological aspects of human brain cysticercosis, in Flisser A, Willms K, Laclette JP, et al (eds): Cysticercosis: Present State of Knowledge and Perspectives: Proceedings of an International Workshop on Cysticercosis Held in San Miguel de Allende, Guanajuato, Mexico, on November 16-18, 1981. New York: Academic Press, 1982, pp 179-200

29. Richards F Jr, Schantz PM: Laboratory diagnosis of cysticercosis. Clin Lab Med 11:1011-1028, 1991

30. Robles C: Consideraciones acerca de 100 casos de tumor cerebral operados. Prensa Med Mex 9:7-79, 1944

31. Rodrigez-Carbajal J, Boleaga-Duran B: Neuroradiology of human cysticercosis, in Flisser A, Willms K, Laclette JP, et al (eds): Cysticercosis: Present State of Knowledge and Perspectives: Proceedings of an International Workshop on Cysticercosis Held in San Miguel de Allende, Guanajuato, Mexico, on November 16-18, 1981. New York: Academic Press, 1982, pp 139-162

32. Rodriguez-Carbajal J, Palacios E, Zee CS: Neuroradiology of cysticercosis of the central nervous system, in Palacios E, Rodriguez-Carbajal J, Taveras JM (eds): Cysticercosis of the Central Nervous System. Springfield, IL: Charles C Thomas, 1983, pp 101-143

33. Santin G, Vargas J: Roentgen study of cysticercisis of the central nervous system. Radiology 86:520-528, 1966

34. Schultz TS, Ascherl GF Jr: Cerebral cysticercosis: occurrence in the immigrant population. Neurosurgery 3:164-169, 1978

35. Shanley JD, Jordan MC: Clinical aspects of CNS cysticercosis. Arch Intern Med, 140:1309-1313, 1980

36. Sotelo J, Escobedo F, Rodriguez-Carbajal J, et al: Therapy of parenchymal brain cysticercosis with praziquantel. N Engl J Med 310:1001-1007, 1984

37. Sotelo J, Guerrero V, Rubio F: Neurocysticercosis: a new clas- sification based on active and inactive forms. A study of 753 cases. Arch Intern Med 145:442-445, 1985

38. Sotelo J, Torres B, Robio-Donnadieu F, et al: Praziquantel in the treatment of neurocysticercosis: long-term follow-up. Neurology 35:752-755, 1985

39. Stern WE: Neurosurgical considerations of cysticercosis of the central nervous sytem. J Neurosurg 55:382-389, 1981

40. Suss RA, Maravilla KR, Thompson J: MR imaging of intracranial cysticercosis: comparison with $\mathrm{CT}$ and anatomopathologic features. AJNR 7:235-242, 1986

41. Takayanagui OM, Jardim E: Aspectos clínicos da neurocisticercose: análise de 500 casos. Arq Neuropsiquiatr 41:50-63, 1983

42. Teitelbaum GP, Otto RJ, Lin M, et al: MR imaging of neurocysticercosis. AJR 153:857-866, 1989

43. Torrealba G, Del Villar S, Tagle P, et al: Cysticercosis of the central nervous system: clinical and therapeutic considerations. J Neurol Neurosurg Psychiatry 47:784-790, 1984

44. Tsang VC, Brand JA, Boyer AE: An enzyme-linked immunoelectrotransfer blot assay and glycoprotein antigens for diagnosing human cysticercosis (Taenia solium). J Infect Dis: 159:50-59, 1989

45. Wilson M, Bryan RT, Fried JA, et al: Clinical evaluation of the cysticercosis enzyme-linked immunoelectortransfer blot in patients with neurocysticercosis. J Infect Dis 164:1007-1009, 1991

46. World Health Organization: Guidelines for Surveillance, Prevention, and Control of Taeniasis/Cysticercosis. Geneva: WHO, 1983, pp 29-82

47. Yang SY, Wang ML, Xue QC: Cerebral cysticercosis. Surg Neurol 34:289-293, 1990

48. Zavala JT: Etiology of cysticercosis, in Palacios E, RodriguezCabajal J, Taveras JM (eds): Cysticercosis of the Central Nervous System. Springfield, IL: Charles C Thomas, 1983, pp $18-26$

49. Zee CS, Segall HD, Apuzzo MLJ, et al: Intraventricular cysticercal cysts: further neuroradiologic observations and neurosurgical implications. AJNR 5:727-730, 1984

50. Zee CS, Segall HD, Boswell W, et al: MR imaging of neurocysticercosis. J Comput Assist Tomogr 12:927-934, 1988

51. Zenteno-Alinis GH: A classification of human cysticercosis, in Flisser A, Willms K, Laclette JP, et al (eds): Cysticercosis: Present State of Knowledge and Perspectives: Proceedings of an International Workshop on Cysticercosis Held in San Miguel de Allende, Guanajuato, Mexico, on November 16-18, 1981. New York: Academic Press, 1982, pp 107-126

Manuscript received April 19, 2002.

Accepted in final form June 5, 2002.

Address reprint requests to: J. Patrick Johnson, M.D., Cedars-Sinai Institute for Spinal Disorders, 444 South San Vicente Boulevard, Suite 800, Los Angeles, California 90048. email: johnsonjp@ cshs.org. 